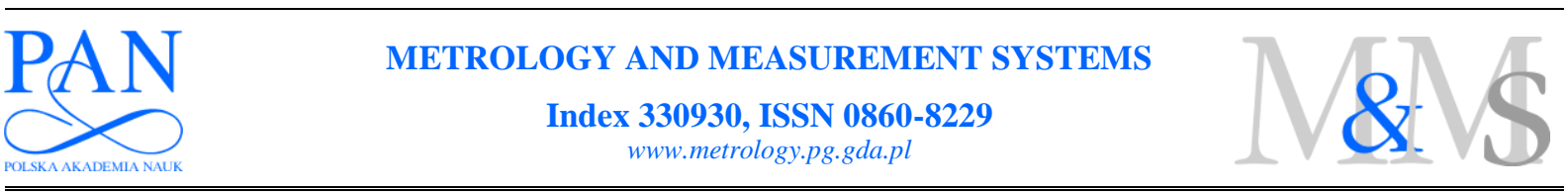

\title{
ALGORITHMS AND METHODS FOR ANALYSIS OF THE OPTICAL STRUCTURE FACTOR OF FRACTAL AGGREGATES
}

\author{
Janusz Mroczka ${ }^{1)}$, Mariusz Woźniak ${ }^{1)}$, Fabrice R.A. Onofri ${ }^{2)}$ \\ 1) Wroclaw University of Technology, Chair of Electronic and Photonic Metrology, ul. B. Prusa 53/55, 50-317 Wroctaw, Poland \\ (janusz.mroczka@pwr.wroc.pl,+487132062 32,هmariusz.wozniak@pwr.wroc.pl,+487132062 25) \\ 2) IUSTI-UMR CNRS 7343, Aix-Marseille University, 5 rue Enrico Fermi, 13453 Cedex 13, Marseille, France \\ (fabrice.onofri@polytech.univ-mrs.fr, +33491106 892)
}

\begin{abstract}
We introduce numerical methods and algorithms to estimate the main parameters of fractal-like particle aggregates from their optical structure factor (i.e. light scattering diagrams). The first algorithm is based on a direct and simple method, but its applicability is limited to aggregates with large size parameter and intermediate fractal dimension. The second algorithm requires to build calibration curves based on accurate particle agglomeration and particle light scattering models. It allows analyzing the optical structure factor of much smaller aggregates, regardless of their fractal dimension and the size of the single particles. Therefore, this algorithm as well as the introduction of a criterial curve to detect the different scattering regimes, are thought to be powerful tools to perform reliable and reproducible analysis.
\end{abstract}

Keywords: fractals, aggregates, light scattering, scattering diagrams, structure factor.

(C) 2012 Polish Academy of Sciences. All rights reserved

\section{Introduction}

Light scattering techniques are widely used to characterize aggregates of nanoparticles observed in, or produced by suspensions, aerosols, dusty plasmas, etc. (e.g. [1, 2]). For the characterization of fractal-like particle aggregates, the most powerful approach is based on recording, and afterwards on the analysis of their optical structure factor (OSF) [1]. However, in the literature, the method used to process the OSF is never detailed and evaluated, whatever the processing scheme (as will be shown later on) it strongly influences the quality and reliability of the analysis. Regarding the previous remarks, the aim of the present work was to develop reliable algorithms to estimate in a comprehensive and reproducible way the fractal parameters from the various OSF. The paper is organized as follows. Section 2 shortly reviews basic equations as well as the aggregation model developed to produce synthetic fractal aggregates. The basic relations between the OSF properties and the fractal aggregates parameters are also introduced in this section. Then, section 3 introduces and details the principle of the two newly-introduced algorithms to process the OSF of fractal-like aggregates. Section 4 presents the main results of the numerical study performed to evaluate the advantages and limits of both algorithms, when tested on two totally different particle systems: colloidal suspensions of optically transparent aggregates (silicon dioxide) and clouds of highly absorbing aggregates (carbonaceous soots). Section 5 is an overall conclusion. 


\section{Theoretical background}

\subsection{Mathematical description}

To describe, by a limited number of parameters, the morphology of fractal-like aggregates, the following equation (sometimes called "fractal equation") [3, 4] is used:

$$
n_{p}=k_{f}\left(\frac{R_{g}}{r_{p}}\right)^{D_{f}},
$$

where $n_{p}$ is the number of particles (referenced here as monomers) in the aggregate, $D_{f}$ its fractal dimension, $k_{f}$ the fractal prefactor (also known as the structural coefficient), $R_{g}$ the radius of gyration of the aggregate and $r_{p}$ the mean radius of the single monomers. The radius of gyration is the mean square distance of the monomers from aggregate's centre of mass (which characterizes the spatial distribution of mass within the aggregate):

$$
R_{g}{ }^{2}=\frac{1}{n_{p}} \sum_{n=1}^{n_{p}}\left(\mathbf{r}_{\mathbf{0}}-\mathbf{r}_{\mathbf{n}}\right)^{2}
$$

where $\mathbf{r}_{\mathbf{n}}$ and $\mathbf{r}_{\mathbf{0}}$ are the vectors pointing to the $\mathrm{n}$-th monomer and aggregate's centre of mass respectively. The fractal prefactor $k_{f}$ is a constant extensively discussed in the literature whose value significantly differs between various authors. As an example, for soot aggregates it can vary from 1.23 to 3.5 (e.g. [5-8]) depending on the particular combustion conditions and miscellaneous techniques used to analyze aggregates. In the present study, like Lapuerta [9] and Sorensen [10] did before, we set for the value of $k_{f}$ the one corresponding to an aggregate of monodisperse monomers with maximum compactness in a 3D space, i.e. an aggregate with compact hexagonal structure:

$$
k_{f}=(\pi / 3 \sqrt{2})(5 / 3)^{3 / 2} \approx 1.593
$$

As an example, Fig. 1 (a) shows a 3D visualization (created with the POV-Ray software [11]) of aggregate defined by $n_{p}=100$ monomers, fractal dimension $D_{f}=1.80$, fractal prefactor $k_{f}=1.593$, radius of gyration $R_{g}=9.97$ and equivalent radius in volume $R_{v}=4.46$. Fig. 1 (b) shows a 2D projection (the image of the aggregate as it is obtained in the zy plane).

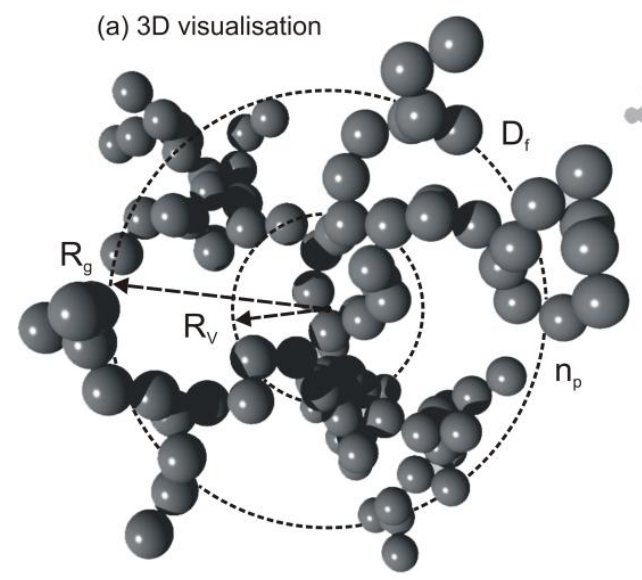

(b) 2D projection

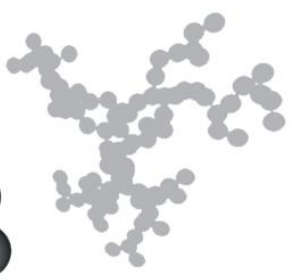

Fig. 1. Numerically generated fractal aggregate having parameters: $n_{p}=100, D_{f}=1.80, k_{f}=1.593, R_{g}=9.97$, $R_{v}=4.64$ (a) 3D rendering with POV-Ray software, (b) $2 \mathrm{D}$ projection of the aggregate. 


\subsection{Numerical code}

To generate synthetic aggregates used in this work, a fully adjustable (tunable) DiffusionLimited-Aggregation (DLA) code has been developed. It allows simulating fractal aggregates of polydisperse, multi-material and overlapping (i.e. partially melted) monomers. Fig. 2 (a) shows an overview diagram of the geometry of the DLA algorithm. In the aggregation process all the primary particles are generated successively at a large distance $R_{p}$ (also called appearance sphere) from the centre of mass of the aggregating cluster:

$$
R_{p} \gg R_{g, n_{p}},
$$

where $R_{g, n_{p}}$ describes the temporary radius of gyration of the growing aggregate. If during its random walk the new particle moves out of the external boundary sphere with radius $R_{e}$, the particle is rejected and another particle is generated at the distance $R_{p}$. The definition of the boundary sphere with radius $R_{e}$, with $\mathrm{R}_{\mathrm{e}} \geq R_{p}$ (ideally $\mathrm{R}_{\mathrm{e}}>>R_{p}$ ) is necessary to avoid particle's roaming far from the aggregate since this will significantly increase computational time. It is important to notice that in the developed numerical code the radiuses of appearance and external boundary spheres are not fixed. These values are continuously optimized. To do so, they are calculated as the sum of the additional constants ( $p$ and $b$ for the appearance and the boundary spheres respectively) and multiply by the radius of the minimum bounding sphere by a factor called "appearance sphere multiplier". This procedure provides a wide range of possible relations between $R_{b}, R_{p}$ and $R_{e}$. For example, it is possible to turn off the multiplication and use only a constant difference between radiuses of the defined spheres.

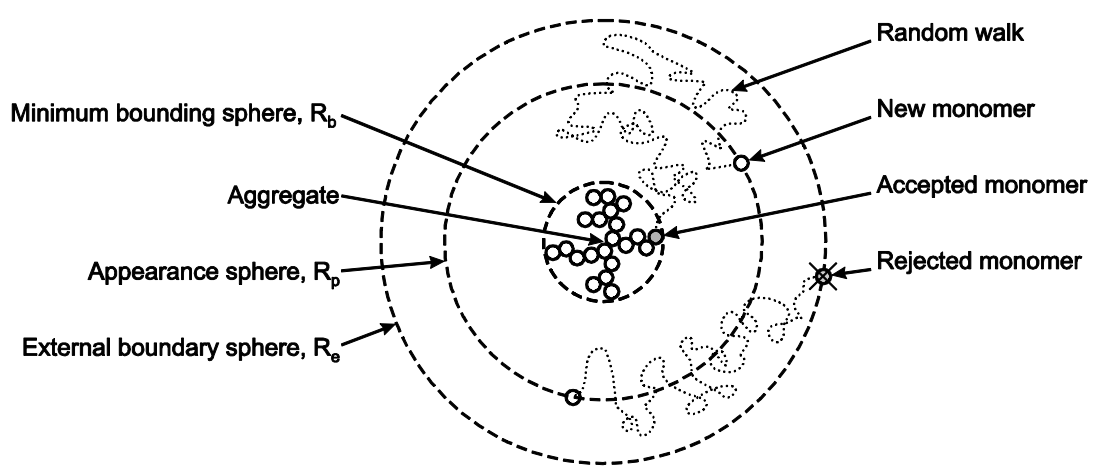

(a)

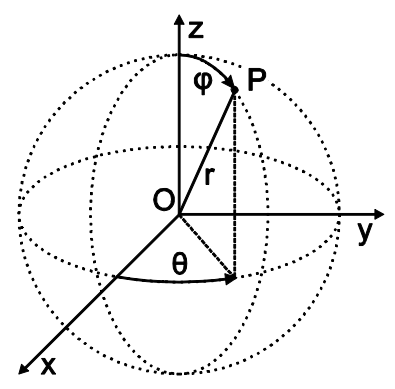

(b)

Fig. 2. (a) Schematic diagram of the Diffusion Limited Aggregation (DLA) steps and parameters and (b) Spherical Coordinate System.

Fig. 2 (b) presents the Spherical Coordinate used in the DLA-type code created in this work. To avoid problems related with temporary position of the growing cluster at each step of the algorithm, the centre of mass of the aggregate is relocated at the centre of the coordinate system. When the aggregation procedure is completed it is necessary to convert the spherical coordinates $\langle r, \theta, \varphi\rangle$ to the classical laboratory Cartesian Coordinate System $<x, y, z>$.

To simulate the random motion of the primary particles, their trajectories were decomposed into small step increments (i.e. $2 r_{p}$ in this study) with a statistically true isotropic orientation. The latter was obtained by generating, at each step, random inclination and azimuth angles $\theta, \varphi$ with a uniform spherical distribution (providing uniformly distributed points on the surface of a sphere) [12]: 


$$
\theta=2 \pi \delta_{1} ; \varphi=2 \arcsin \sqrt{\delta_{2}},
$$

where $\delta_{1}$ and $\delta_{2}$ have uniform distributions on [0-1]. Finally, if collision between a marching monomer and the aggregate occurs, criteria for irreversible aggregation should be provided. Thus, after the impact between a single monomer and an aggregate of $n_{p}-1$ particles, the sticking process is effective only when the following conditions are satisfied:

$$
\left(\frac{R_{g, n_{p}}}{R_{g, n_{p}-1}}\right)^{D_{f}-\varepsilon} \leq \frac{n_{p}}{n_{p}-1} \leq\left(\frac{R_{g, n_{p}}}{R_{g, n_{p}-1}}\right)^{D_{f}+\varepsilon},
$$

where $\varepsilon$ is an accuracy parameter on the fractal dimension. In the DLA software this value may be adjusted on demand. Nevertheless, in the present study it was fixed at $\varepsilon=10^{-2}$ as the smaller values greatly increase computational time without noticeable improvements in morphological characteristics of the aggregates. Something important to understand is that Eq. (6) allows to ensure at each aggregation step that Eq. (1) is nearly satisfied and thus, that the scaling properties of all aggregates are conserved at all scales. During the aggregation process we assume that particles stick together like hard spheres in contact, i.e. exactly at one point and any additional displacement after their collision is impossible. This is not necessarily the case for nanoparticles, which are mostly sensitive to adhesion and short range forces (e.g. Van der Waals forces). Nevertheless, because of the complex description and an unknown physical basis, it is common to simplify this problem and neglect the other phenomena $[3,13]$. A more detailed description of the technical functionalities of our tunable DLA code may be found in [14].

\subsection{Light scattering models}

The optical characterization of nanoparticles requires a proper particle model and an accurate light scattering model. The Lorenz-Mie Theory (LMT, [15]) is still widely used to calculate the absorption and scattering properties of spherical homogeneous particles. Its applicability is nevertheless limited to the characterization of spherical particles $[16,17]$. For particle aggregates there are other semi-analytical theories and numerical alternatives, like the T-Matrix method (or null-field method) and the Discrete Dipole Approximation [18]. In the present work, we used the T-Matrix method and more particularly the Fortran code developed by D. W. Mackowski and M. I. Mischenko [19]. This code has been paralleled to allow the calculation of the scattering properties of optically dilute systems of aggregates of nanospheres [20].

\section{4. $\quad$ Light scattering properties}

With linear optics, one of the most fundamental approaches to characterize the size distribution of large particles is to analyze their scattering diagrams, i.e. the scattered intensity $I$ versus the scattering angle $\theta$ (e.g. in the Fraunhofer, the rainbow or critical angle regions [17]). To characterize nano- and micro-aggregates the scattered intensity must be analyzed over a wide angular range and it is better to analyze its angular dependency with respect to the amplitude of the scattering vector $q$ :

$$
q=\frac{4 \pi}{\lambda} \sin \left(\frac{\theta}{2}\right)
$$

where $\lambda$ is the incident wavelength. The quantity $I(q)$ is usually referred as the "optical 
structure factor (OSF)" [1]. Fig. 3 (a) shows the numerical simulation of the evolution of the OSF of a water suspension of silicon dioxide aggregates. Results have been averaged over 500 different aggregates with parameters: $n_{p}=100, D_{f}=1.80, r_{p}=55 \mathrm{~nm}, R_{g}=550 \mathrm{~nm}$ and $\tilde{m}_{p}=1.47+0 i$ (where $\tilde{m}_{p}$ is the complex refractive index of $\mathrm{SiO}_{2}$ [21]). Three scattering regimes (or zones) may be indentified in this OSF: namely, the Guinier, the fractal (or powerlaw) and the Porod zones. Behavior of the OSF in each zone is totally different and related to different aggregate properties. The OSF in the Guinier zone is essentially dependent on the overall size of aggregates (i.e. the length of this zone, with respect to the scattering vector, gives information about $R_{g}$ ). In the fractal zone, the power law decay of the OSF (and the associated slope) depends mostly on the aggregate's morphology and thus, $D_{f}$. The behavior of the OSF in the Porod zone is known to be mainly sensitive to monomers size [1].

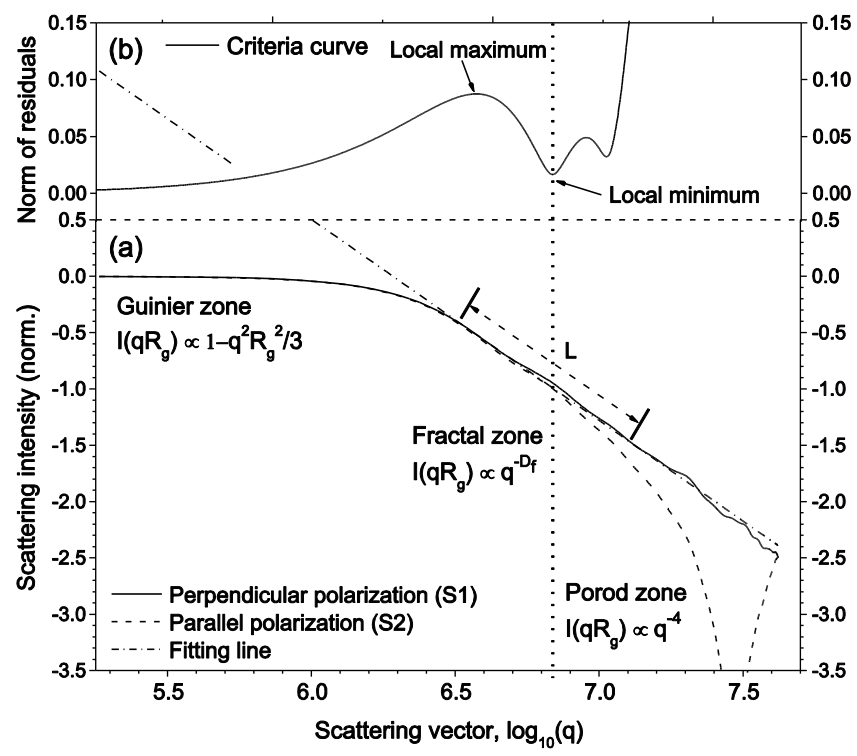

Fig. 3. (a) Evolution of the optical structure factor (OSF) of silicon dioxide aggregates $\left(n_{p}=100, D_{f}=1.80\right.$, $r_{p}=55 \mathrm{~nm}, R_{g}=550 \mathrm{~nm}, \tilde{m}_{p}=1.47+0 i$ ) and (b) criteria curve obtained with the FSE algorithm.

\section{Material and methods}

\subsection{Estimation of the radius of gyration}

We basically use the Guinier equation [1] to estimate the radius of gyration from the normalized OSF (where $I(q)$ is the scattered intensity):

$$
\frac{I(q=0)}{I(q)} \approx 1+\frac{R_{g}{ }^{2} q^{2}}{3}, \quad \text { for } \quad q R_{g}<1 .
$$

In the previous equation the power-law term indicates that from the normalized OSF plotted versus $q$ we can estimate directly the radius of gyration. To do so, the algorithm works in a two-step process. Firstly, the OSF is low-pass filtered and normalized. Secondly, a linear least square fitting method is used to estimate the related slope coefficient in the Guinier zone, symbolized here by the parameter $a$ and its related accuracy $\Delta a$. Finally, the radius of gyration is evaluated as $R_{g}=\sqrt{3 a}$, with:

$$
\Delta R_{g} \approx \frac{\partial R_{g}}{\partial a} \Delta a=\frac{3}{2 \sqrt{3 a}} \Delta a .
$$




\subsection{Estimation of the fractal dimension}

\subsubsection{Second Slope Estimation (SSE) algorithm}

In the fractal zone, the power-law decay of the OSF provides both quantitative and qualitative information about the fractal dimension of the aggregates. To get directly the value of the $D_{f}$, only the data $q R_{g}>5$ must be used [1]. In fact, below this limit a true power-law regime is not clearly observed. Like previously done for the radius of gyration, the fractal dimension can be obtained directly from the slope coefficient estimated with a linear least square fitting method. However, this procedure and the related algorithm are only valid for rather large aggregates, i.e. $R_{g}>(5 \lambda) /(4 \pi)$.

On the other hand to get correct estimation of $D_{f}$, a minimum number of data points are necessary and they must also cover a minimum scattering angles range. Numerical simulations, not reported here, have shown that the latter criterion must be reinforced at least by a factor of two, $R_{g}>(5 \lambda) /(2 \pi)$ and that the predictions obtained are only satisfactory for fractal-like aggregates whose dimensions are restricted to the range $D_{f}=1.6-2.0$.

\subsubsection{First Slope Estimation (FSE) algorithm}

The "First Slope Estimation (FSE)" algorithm was developed to process OSF with a scattering vector that do not necessarily satisfies the $q R_{g}>5$ condition. The biggest advantage of the second algorithm, which uses calibration curves, is its applicability to all kind of aggregates, regardless their fractal dimension, wavelength or radius of gyration. This algorithm works in a five-step process.

\section{A. Signal (optical structure factor) interpolation.}

To compensate the huge range of variation of the scattering vector and its non-linearity with respect to the scattering angle (which both induce a non constant sampling rate of the optical structure factor), the OSF is resampled with a linear interpolation scheme. The number of points in the interpolation procedure is chosen arbitrarily, as a compromise between accuracy and algorithm execution time. Basically, it is set to 1000 points per one order of magnitude of $q$. So that, depending on the incident wavelength, the OSF is sampled in 20003000 data points.

\section{B. Linear least square fitting.}

One of the most important parts of the FSE algorithm relies on the construction and analysis of a set of criteria curves. The latter uses a local linear least square (LLS) fitting procedure of the OSF. To do so, at each iteration step of the algorithm, a moving window with fixed length analyzes the local slope of the OSF. The LLS is performed according to the minimization procedure described by the equation:

$$
\min _{x}\|\mathbf{A} \mathbf{x}-\mathbf{b}\|_{2}, \quad \mathbf{A} \in R^{m \times n},
$$

where $\mathbf{x}$ represents the experimental vector containing windowed data of the OSF and $\mathbf{A}, \mathbf{b}$ are matrixes containing regression coefficients. At the beginning of the algorithm, the length of the initial window (usually 600 points) is set to a relatively large value and it may be changed depending on the criteria described at point $\mathrm{C}$. 


\section{Criteria curve construction.}

For each fitting step, and as a measure of the solution quality, the norm of the overall residual is calculated. Looking for the minimum residual corresponding to each position of the analysis windows, a criteria curve is built. It gives information about fitting results in each part of the optical structure factor. Fig. 3 (b) shows the best fitting line and criteria curve corresponding to the analysis of the OSF displayed in Fig. 3 (a). It is easy to notice that the norm of the residuals of Eq. (10) varies depending on the window position with respect to the scattering vector, i.e. the scattering regimes.

It is close to zero in the Guinier zone, and it is significantly increasing during transition between the Guinier and Fractal zones. In the same way, the central part of the fractal zone is well identified by a local minimum. This local minimum is defined as the one that just follows the first local maximum or increasing $q$. The latter maximum identifies the transition between the Guinier and fractal zones. Fig. (3) (b) shows also that in the Porod zone, the criteria curve is continuously increasing (i.e. the size parameter of these aggregates is too small to allow the observation of the Porod scattering regime). Note that, depending on the width used for the analysis windows, the shape and behavior of the criteria curve may be slightly different. The polarization state of the incoming plane wave influences also the shape of the criteria curve but, in all calculation presented here, we use the perpendicular one since it is more appropriate to detect the fractal regime.

\section{Analysis of the criteria curve}

To find the best solution from the fitting procedure, it is necessary to select the right local minimum of the criteria curve. An additional criterion is used for this purpose. This local minimum must satisfy the "absolute value criterion" i.e. the absolute value of the minimum point is at least two times smaller than the value of the first maximum.

\section{E. Algorithm iteration}

If the local minimum detected at the previous step does not satisfy the "absolute value criterion", the entire procedure (points B-D) is repeated for smaller and smaller windows widths. If after several iterations this criterion is still not satisfied, the algorithm stops. It usually means that the OSF does not exhibit any characteristic fractal zone. This situation occurs for small size parameter aggregates (i.e. $\pi R_{g} / \lambda<1 / 2$ ). Typically, as a limit value that stops the algorithm, a length of the window 10 times smaller than the initial value is taken (i.e. 60 points of the OSF).

\section{Results and discussion}

For computational efficiency, we built an extensive database of numerical (synthetic) aggregates as well as of their scattering properties. To demonstrate the validity of the algorithm introduced above, two typical particle systems and parameters were considered:

- An aqueous colloidal suspension of aggregates, composed of silicon dioxide $\left(\mathrm{SiO}_{2}\right)$ nanobeads with radius $r_{p}=55 \mathrm{~nm}$ and refractive index $\tilde{m}_{p}=1.47+0 i$ [21] and with $R_{g}=110-550 \mathrm{~nm}$ (with step equal to $11 \mathrm{~nm}$ ), fractal dimension $D_{f}=1.4,1.6, \ldots, 2.8$, $n_{p}=4-1000$ (according to the other parameters, see Eq. (1)). The probing beam is a local plane wave with perpendicular polarization and nominal wavelength $\lambda=409 \mathrm{~nm}$ (violet laser diode).

- A gas flow of soot aggregates, composed of carbonaceous monomers with radius $r_{p}=25 \mathrm{~nm}$, refractive index $\tilde{m}_{p}=1.71+0.56 i[22], R_{g}=188-1625 \mathrm{~nm}, D_{f}=1.4,1.6, \ldots, 2.8$ 
and $n_{p}=450,500$ and 550. The probing beam is a local plane wave with perpendicular polarization and nominal wavelength $\lambda=532 \mathrm{~nm}$ (frequency doubled Nd-YAG laser).

For all the numerical results presented here, to cancel out orientation effects, scattering diagrams have been averaged over 500 different aggregates with exactly the same fractal properties but with random orientation.

\subsection{Estimation of the radius of gyration}

To estimate the radius of gyration of the silicon dioxide and soot aggregates, the Guinier zone is first analyzed. Fig. 4 presents the results of the analysis of 5 colloidal suspensions containing aggregates with increasing radiuses of gyration. The estimated radiuses of gyration were found to be 236, 296, 356, 418 and $595 \mathrm{~nm}$ for the initial values 220, 275, 330, 385 and $550 \mathrm{~nm}$ respectively. Note that linear regression fits really well the data points (regression coefficient equals $0.9985,0.9986,0.9986,0.9984$ and 0.9983 respectively), even if all the estimated values are slightly, but systematically, overestimated.

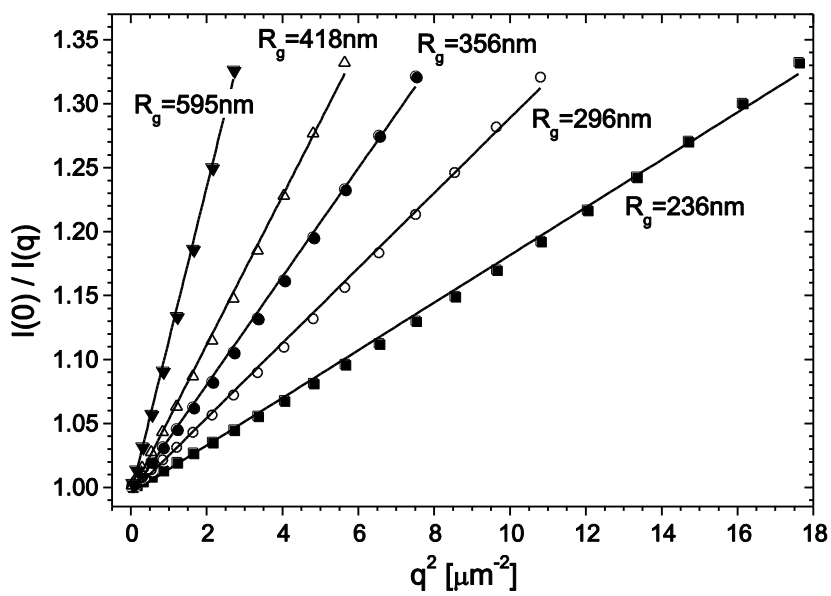

Fig. 4. Guinier analysis of the selected $\mathrm{SiO}_{2}$ aggregates.

Table 1 resumes the parameters estimated for different systems of soot aggregates. Here also the estimated values fit pretty well the nominal ones (i.e. initially imposed during the aggregation). The overestimation of the radius of gyration evolves from 1 to $9 \%$ for $D_{f} \leq 2.0$.

Table 1. Summary of the radiuses of gyration estimated for soot aggregates of $n_{p}=500$ monomers.

\begin{tabular}{|c|c|c|c|c|c|c|c|c|}
\hline Fractal dimension & 1.4 & 1.5 & 1.6 & 1.7 & 1.8 & 1.9 & 2.0 & 2.1 \\
\hline Initial $\mathrm{R}_{\mathrm{g}}[\mathrm{nm}]$ & 1518 & 1155 & 909 & 736 & 610 & 515 & 443 & 386 \\
\hline Estimated $\mathrm{R}_{\mathrm{g}}[\mathrm{nm}]$ & 1541 & 1167 & 954 & 794 & 662 & 540 & 483 & 425 \\
\hline $\mathrm{R}_{\mathrm{g}}$ accuracy $[\mathrm{nm}]$ & 73 & 53 & 50 & 41 & 34 & 26 & 23 & 20 \\
\hline Fractal dimension & 2.2 & 2.3 & 2.4 & 2.5 & 2.6 & 2.7 & 2.8 & \\
\hline Initial $\mathrm{R}_{\mathrm{g}}[\mathrm{nm}]$ & 341 & 304 & 274 & 250 & 229 & 211 & 195 & \\
\hline Estimated $\mathrm{R}_{\mathrm{g}}[\mathrm{nm}]$ & 380 & 343 & 312 & 288 & 270 & 255 & 241 & \\
\hline $\mathrm{R}_{\mathrm{g}}$ accuracy $[\mathrm{nm}]$ & 17 & 16 & 14 & 12 & 11 & 11 & 10 & \\
\hline
\end{tabular}




\subsection{Estimation of the fractal dimension}

Fig. 5 presents results obtained for the fractal dimension of soot aggregates, with initial fractal dimension $D_{f}=1.8$ and $n_{p}=250$ monomers. The fractal dimension estimated with the SSE algorithm, $D_{f}=1.82 \pm 0.01$ is in a very good agreement with the expected one. However, as already mentioned, it must be kept in mind that the applicability of the SSE method is limited to OSF with $q R_{g}>5$.

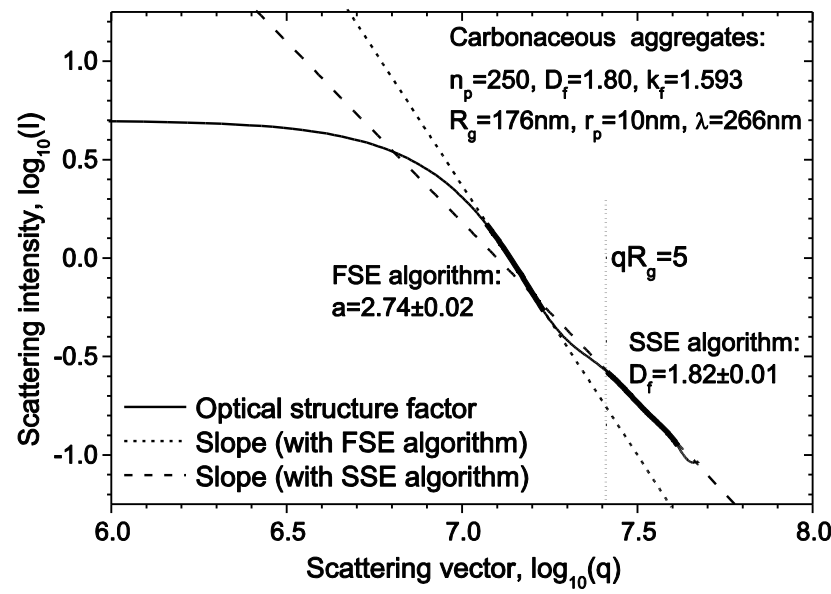

Fig. 5. Comparison between the SSE and raw FSE (without calibration curves) algorithms for soot aggregates with initial fractal dimension $D_{f}=1.8, r_{p}=10 \mathrm{~nm}$ and $n_{p}=250$.

Table 2 summarizes the fractal parameters estimated with the SSE algorithm for the soot aggregates with increasing number of monomers. Note that with the SSE algorithm is was impossible to proceed smaller aggregates than those considered in this table (i.e. due to $q R_{g}>5$ limitation). In an opposite way, Fig. 5 shows also that the raw slope found in the fractal zone, by the FSE algorithm $2.74 \pm 0.02$, differs significantly from the expected fractal dimension.

Table 2. SSE algorithm: estimation of the radius of gyration and fractal dimension of soot aggregates.

\begin{tabular}{l|cccccc}
\hline Number of monomers $n_{p}$ & 160 & 180 & 200 & 220 & 240 & 242 \\
\hline Radius of gyration $R_{g}[\mathrm{~nm}]$ & 127.6 & 137.4 & 146.1 & 155.5 & 163.6 & 172.1 \\
\hline Initial $D_{f}$ & 1.80 & 1.80 & 1.80 & 1.80 & 1.80 & 1.80 \\
\hline Estimated $D_{f}$ & 1.997 & 1.941 & 1.838 & 1.812 & 1.772 & 1.795 \\
\hline \multicolumn{7}{|c|}{} \\
\hline Number of monomers $n_{p}$ & 246 & 246 & 248 & 249 & 250 \\
\hline Radius of gyration $R_{g}[\mathrm{~nm}]$ & 172.1 & 173.9 & 174.8 & 175.3 & 175.7 \\
\hline Initial $D_{f}$ & 1.80 & 1.80 & 1.80 & 1.80 & 1.80 \\
\hline Estimated $D_{f}$ & 1.802 & 1.818 & 1.828 & 1.829 & 1.831 \\
\hline
\end{tabular}

Fig. 6 shows the fractal dimension estimated with the FSE algorithm for silicon dioxide aggregates with the radius of gyration increasing from 110 to $550 \mathrm{~nm}$ and initial fractal dimensions (a) 1.80 and (b) 2.20. From this figure and complementary results, we derived 
calibration curves for the FSE algorithm that relate the raw slope associated to the fractal zone with the aggregates fractal dimension and gyration radiuses, see Fig. 7.

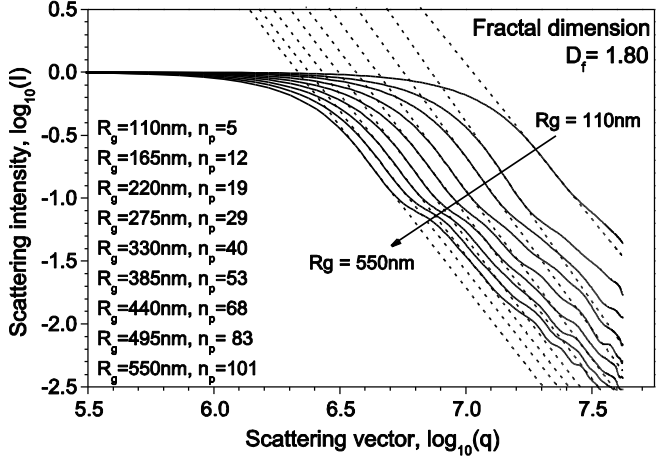

(a)

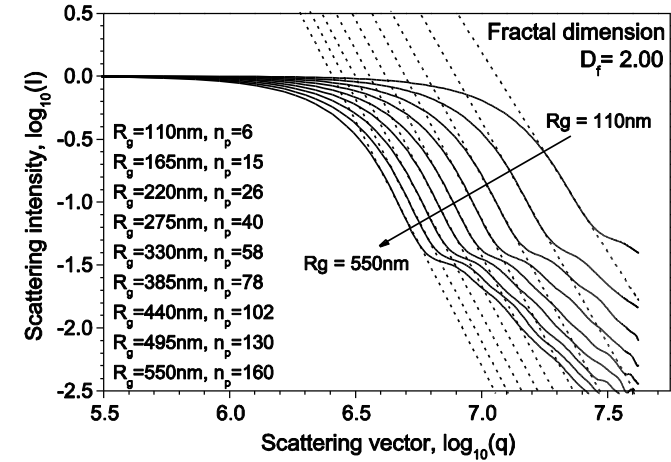

(b)

Fig. 6. Typical results for the fractal dimension estimated for $\mathrm{SiO}_{2}$ particle aggregates with nominal fractal dimension: (a) 1.80, and (b) 2.00 .

A 3rd order polynomial fitting improves the resolution of these calibration curves. Note that in Fig. 7 all the curves are almost superimposed for aggregates with a radius of gyration higher than $6 r_{p}$, i.e. the fractal dimension of large aggregates can be determined without any prior knowledge (or analysis) of the radius of gyration.

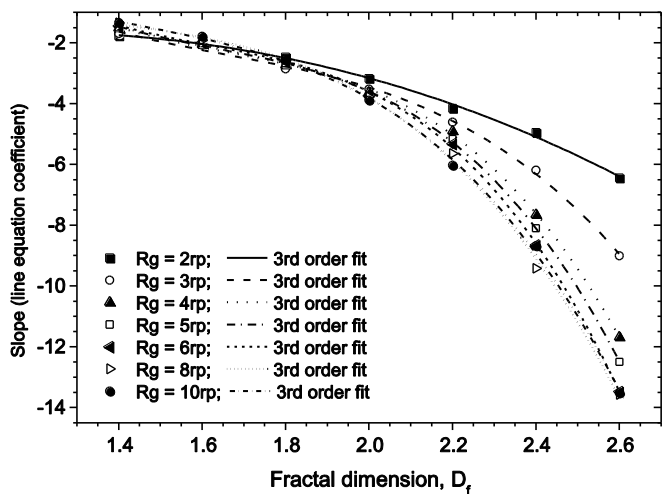

Fig. 7. Estimated slope as a function of the fractal dimension for silicon dioxide aggregates (the FSE algorithm).

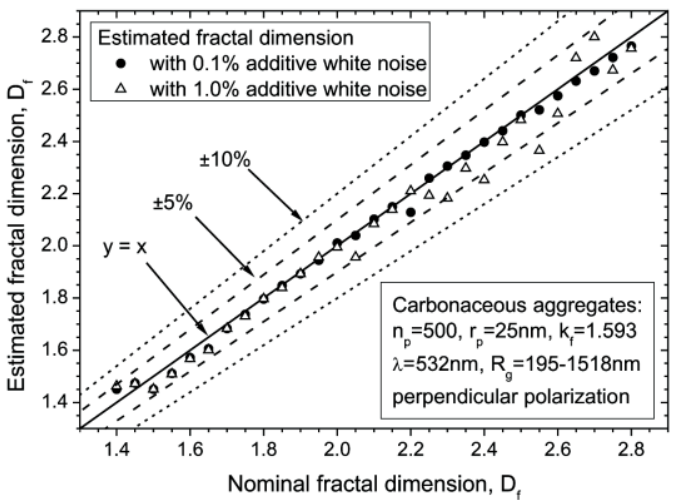

Fig. 8. FSE algorithm with calibration curves: automatic estimation of the fractal dimensions from noisy optical structure factors. Parameters: soot aggregates with fractal dimension from 1.40 to 2.80 and constant number of 500 monomers. 
Fig. 8 shows that by combining the FSE algorithm and the calibration curves (like the ones presented in Fig. 7), the estimation of the aggregates fractal dimension is significantly improved even when $0.1 \%$ and $1 \%$ of white noise is added to the OSF. In fact, the fractal dimension is estimated over a large range and with an accuracy $5 \%$ with $0.1 \%$ of additive white noise and about $10 \%$ for $1 \%$ of additive white noise.

\section{Conclusion}

We introduced a modeling procedure and numerical algorithms to estimate the fractal dimension (and radius of gyration) of fractal-like aggregates from the analysis of their optical structure factor. All numerical results provided here are based on the accurate particle agglomeration and light scattering models. The Second Slope Extraction (SSE) algorithm is rigorously based on the Guinier analysis. It provides a simple and direct estimation of the fractal dimension of the investigated aggregates. However, its applicability is limited to largesize parameter aggregates. The First Slope Extraction (FSE) algorithm can be used to analyze much smaller aggregates than the SSE algorithm, even for values of the optical structure factor below $q R_{g}=5$. Its application is more complicated than the SSE algorithm, as it requires to calculate look-up tables and to define calibration curves. However, the FSE algorithm is applicable to all kinds of aggregates, regardless of their morphology. Therefore, it is thought to be a much more powerful and universal solution. The criteria curves is also a useful approach to indentify the different scattering regimes and to obtain more reproducible results.

\section{References}

[1] Sorensen, C.M. (2001). Light Scattering by Fractal Aggregates: A Review. Aerosol Science and Technology, 35, 648-687.

[2] Onofri, F.R.A., Woźniak, M., Barbosa, S. (2011). On the Optical Characterization of Nanoparticle and their Aggregates in Plasma Systems. Contributions to Plasma Physics, 51(2-3), 228-236.

[3] Witten, T.A., Sander, L.M. (1981). Diffusion-Limited Aggregation, a Kinetic Critical Phenomenon. Physical Review Letters, 47(19), 1400-1403.

[4] Jullien, R., Botet, R. (1987). Aggregation and fractal aggregates. Singapore: World Scientific.

[5] Cai, J., Lu, N., Sorensen, C.M. (1995). Analysis of Fractal Cluster Morphology Parameters: Structural Coefficient and Density Autocorrelation Function Cutoff. Journal of Colloid and Interface Science, 171(2), 470-473.

[6] Koylu, U.O., Faeth, G.M., Farias, T.L., Carvalho, M.G. (1995). Fractal and Projected Structure Properties of Soot Aggregates. Combustion and Flame, 100(4), 621-633.

[7] Puri, R., Richardson, T.F., Santoro, R.J., Dobbins, R.A. (1993). Aerosol Dynamic Processes of Soot Aggregates in a Laminar Ethene Diffusion Flame. Combustion and Flame, 92(3), 320-333.

[8] Ouf, F.X., Yon, J., Ausset, P., Coppalle, A., Maille, M. (2010). Influence of Sampling and Storage Protocol on Fractal Morphology of Soot Studied by Transmission Electron Microscopy. Aerosol Science and Technology, 44(11), 1005-1017.

[9] Lapuerta, M., Martos, F.J., Martín-Gonzalez, G. (2009). Geometrical determination of the lacunarity of agglomerates with integer fractal dimension. Journal of Colloid and Interface Science, 346(1).

[10] Sorensen, C.M. (1997). Light Scattering by Fractal Aggregates: A Review. Aerosol Science and Technology, 35, 648-687.

[11] POV-Ray, Persistence of Vision Pty. Ltd., 3.6 ed: Persistence of Vision Raytracer (Version 3.6) [Computer Software], 2004, Retrieved 28.02.2009 from http://www.povray.org/download.

[12] Feller, W. (1971). An Introduction to Probability Theory and Its Applications, 2, New York. 
[13] Babu, S., Gimel, J.C., Nicolai, T. (2008). Diffusion limited cluster aggregation with irreversible slippery bonds. The European Physical Journal E, 27(3).

[14] Woźniak, M., Onofri, F.R.A., Barbosa, S., Yon, J., Mroczka, J. (2012). Comparison of methods to derive morphological parameters of multifractal samples of particle aggregates from TEM images. Journal of Aerosol Science, 47, 12-26.

[15] Mie, G. (1908). Beiträge zur Optik trüber Medien, speziell kolloidaler Metallösungen. Annalen der Physik, 330, 377-445.

[16] Bohren, C.F., Huffman, D.R. (1998). Absorption and Scattering of Light by Small Particles. John Wiley and Sons, Inc.

[17] Xu, R. (2002). Particle Characterization: Light Scattering Methods. New York: Kluwert Academic Publisher.

[18] Draine, B.T., Flatau, P.J. (2010). User Guide for the Discrete Dipole Approximation Code DDSCAT 7.1. Instrumentation and Methods for Astrophysics.

[19] Mishchenko, M., Travis, L.D., Mackowski, D.W. (2012). T-matrix Codes for Computing Electromagnetic Scattering by Nonspherical and Aggregated Particles. Retrieved 1.04.2009, from http://www.giss.nasa.gov/staff/mmishchenko/t_matrix.html.

[20] Mishchenko, M., Travis, L.D. (1998). Capabilities and limitations of a current Fortran implementation of the T-Matrix method for randomly oriented, rotationally oriented symmetric scatters. Journal of Quantitative Spectroscopy and Radiative Transfer, 60(3), 309-324.

[21] Sopra.S.A. (2010). Optical Data from Sopra S.A. Refractive Indexes Database: http://www.sopra-sa.com.

[22] Tourbin, M. (2006). Caracterisation comportement de suspensions concentrees de nanoparticules sous ecoulement: application aux processus d'agregation et de rupture. PhD Toulouse, National Polytechnique de Toulouse. 